0031-3203(94)00063-8

\title{
CORNER POINT DETECTION USING NEST MOVING AVERAGE
}

\author{
Soo-Chang Pei and Ji-Hwei Horng \\ Department of Electrical Engineering, National Taiwan University, Taipei, Taiwan, Republic of China
}

(Received 27 July 1993; in revised form 6 April 1994; received for publication 20 May 1994)

\begin{abstract}
Corners are rich in high frequency components. A low-pass filter suppresses corners and results in a smooth curve. Therefore, corner points have a larger amount of shift than other points and can easily be detected by a low-pass filter. Experiments suggest that a moving average can do this job suitably with a low computational load and good locality in spatial domain. A moving average with a smaller size window is used to perprocess the digital boundary with quantization error or noise.
\end{abstract}

Corner detection Nest moving average

\section{INTRODUCTION}

Corner points of a shape's boundary are rich in information. Polygonal approximation based on these points forms a good estimation of the boundary. Thus, the corner point is a very important feature in shape recognition, especially for occluded shape recognition, where boundary segmentation is an important procedure.

In this paper, we propose a corner point detection system, which is robust under a noisy environment. Good locality in the spatial domain is also important, if the application to occluded shape recognition where only local information is useful is to be taken into consideration. Computation time is another factor for concern - it should be as small as possible

\section{DETECTION OF CORNER POINTS}

\section{Frequency domain analysis}

Consider the Fourier descriptor of a shape's boundary. The content of the high-frequency band are due to corners, which are the rapid variations of boundary. The smooth portions of the shape's boundary give rise to the contents of the low-frequency band.

In practical digital images, quantization errors and noise may be present. Their typical size are one or two pixels. Therefore, they have even higher frequency components than typical corners.

\section{The moving average}

Moving average is defined by the equation

$$
y[n]=\frac{1}{2 M+1} \sum_{k=-M}^{M} x[n-k] .
$$

It computes the $n$th point of the output signal as the average of $2 M+1$ points of the input signal centered the $n$th point.
Impulse response and magnitude of frequency response of a moving average is plotted in Fig. 1. The magnitude response falls off at high frequencies. This attenuation of the high frequencies suggests that the system will smooth out rapid variations in the input sequence; that is, it is a rough approximation to a low-pass filter.

The moving average is a FIR filter with good locality in spatial domain. Locality is a very important property in partial shape recognition where only local information is of concern. If part of the shape is occluded or distorted, the moving average of the remaining portion will not be affected by the occlusion or distortion. Another advantage of locality is the reduction of computation load.

\section{Proposed method}

The boundary points can be defined in parametric form

$$
r[n]=x[n]+j y[n] \quad n=1,2, \ldots, N,
$$

where $n$ is the pathlength along the boundary.

Low-pass filtering of the boundary sequence using a moving average filter results in a smooth curve. The smoothing effect of the moving average will shift the corner points by a larger distance than the other points. When calculating the difference between the input and output sequences, corner points will lie on the local maxima of the absolute difference.

The corresponding algorithm is summarized below

\section{Filtering}

$$
r_{0}[n]=\frac{1}{2 M+1} \sum_{k=-M}^{M} r[n-k] \quad n=1,2, \ldots, N,
$$

where $r[n]=r[(n$ modulo $N)]$.

Calculate absolute difference. 

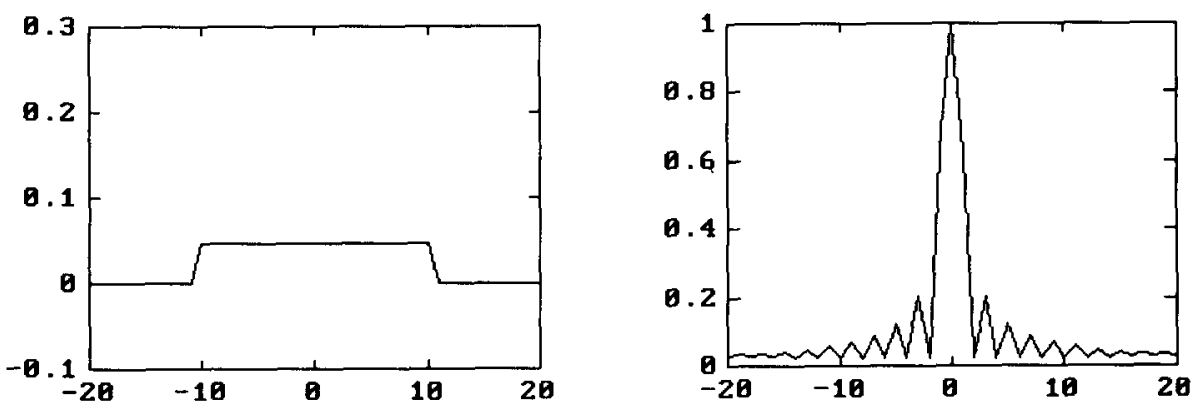

Fig. 1. Impulse and amplitude responses of the moving average.

$$
d[n]=\left|r_{0}[n]-r[n]\right| \quad n=1,2, \ldots, N .
$$

Find local maxima of the sequence $d[n]$, which are corner points.

\section{Robustness considerations}

In practical digital image systems, quantization error and some other noise may be present. These will result in extra local maxima in the absolute difference sequence. To reduce the noise effect, another low-pass filter is required. It should have a suitable passband, in order to suppress the noise power but let along the true signal.

Modification of the proposed method is to add another moving average filter of a proper size to filter the noisy boundary.

The noise reduction filter must have a wider passband than the one used previously, and can be defined by

$$
y[n]=\frac{1}{2 m+1} \sum_{k=-m}^{m} x[n-k],
$$

where $m$ is a constant smaller than $M$. The modified algorithm is described as follows:

\section{Filtering.}

$$
\begin{aligned}
& r_{01}[n]=\frac{1}{2 m+1} \sum_{k=-m}^{m} r[n-k] \quad n=1,2, \ldots, N, \\
& r_{02}[n]=\frac{1}{2 M+1} \sum_{k=-M}^{M} r[n-k] \quad n=1,2, \ldots, N,
\end{aligned}
$$

where $r[n]=r[(n \operatorname{modulo} N)]$.

Calculate the absolute difference.

$$
d[n]=\left|r_{02}[n]-r_{01}[n]\right| \quad n=1,2, \ldots, N .
$$

Find the local maxima of the sequence $d[n]$; those are corner points.

\section{Fast algorithm}

The two filters, which were introduced for different purposes, can be combined into a nest moving average.

Let the impulse response of the original lawpass filter be $h_{M}[n]$ and the impulse response of the noise reduction filter be $h_{m}[n]$. Then, the absolute difference sequence can be calculated as

$$
\begin{aligned}
d[n] & =\left|r[n]^{*} h_{M}[n]-r[n]^{*} h_{m}[n]\right| \\
& =\left|r[n]^{*}\left(h_{M}[n]-h_{m}[n]\right)\right| \\
& =\left|r[n]^{*}\left(h_{m}[n]-h_{M}[n]\right)\right|,
\end{aligned}
$$

where $n=1,2, \ldots, N$. Which is equivalent to calculating the absolute value of the boundary sequence filtered by a filter with impulse response defined by

$$
h[n]=h_{m}[n]-h_{M}[n] .
$$

The impulse response of the nest moving average and its corresponding magnitude response is plotted in Fig. 2. Observe that the nest moving average is a rough approximation to a bandpass filter with nice locality in the spatial domain. The bandpass filter
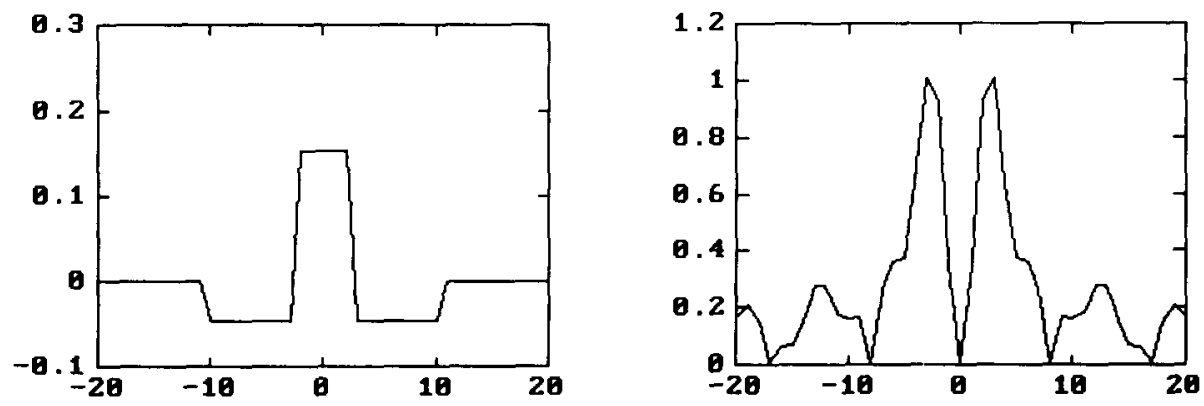

Fig. 2. Impulse and amplitude responses of the nest moving average. 
suppresses the smooth portion of the boundary in the low frequency band and reduces the noise in the high frequency band.

The difference equation of the nest moving average is defined by

$$
\begin{aligned}
d[n] & =\left(\frac{1}{2 m+1}-\frac{1}{2 M+1}\right) \sum_{|k| \leq m} x[n-k] \\
& +\left(-\frac{1}{2 M+1}\right) \sum_{m<|k| \leq M} x[n-\mathrm{k}] .
\end{aligned}
$$

Thus, $2 M$ additions and 2 multiplications are required for the calculation of each output point $d[n]$. For the overall boundary, only $2 M N$ additions and $2 N$ multiplications are required. The computation time is considerably reduced.

\section{EXPERIMENTAL RESULTS}

Figure 3(a) shows the boundary of a wrench (solid line) together with its moving-averaged version (dashed line). The dashed line does not follow the solid line very well as the portions where the solid line changes rapidly. The absolute difference of these two lines is plotted in Fig. 3(b). Local maxima are denoted by cross marks. The corresponding boundary points are marked by small circles in Fig. 3(c). Note that there are some extra points falsely generated due to noise.

Simulation results using the modified algorithm are plotted in Fig. 4. The dash-dot line in Fig. 4(a) is a noise-reduced version of the boundary. The corner points are detected quite well.

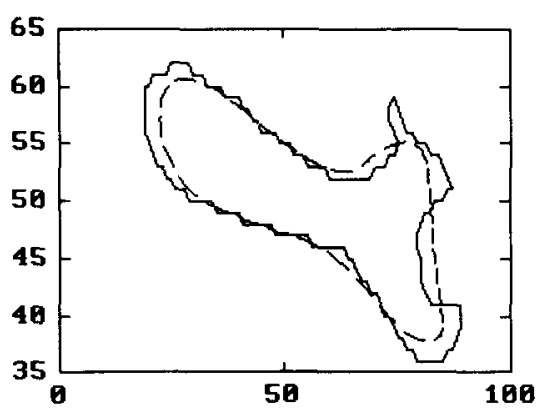

Fig. 3. (a) A wrench and its moving average.

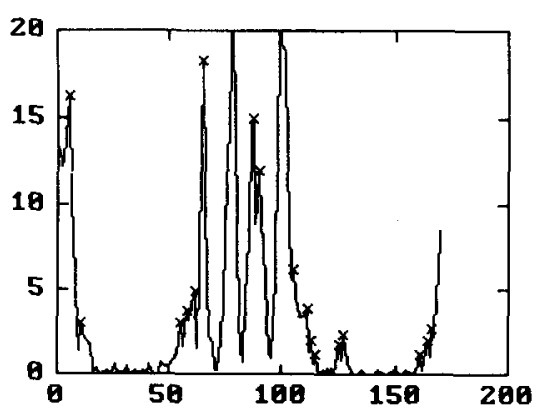

Fig. 3. (b) Local maxima of the absolute difference.

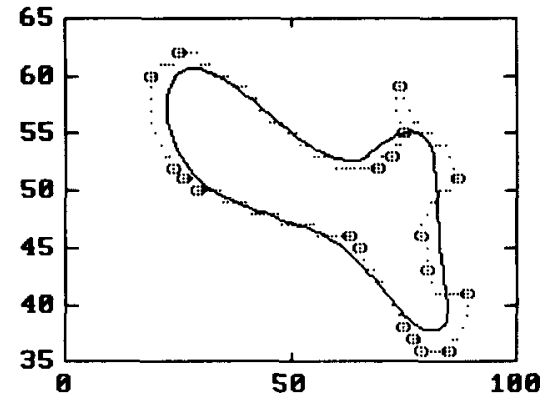

Fig. 3. (c) Corner points.

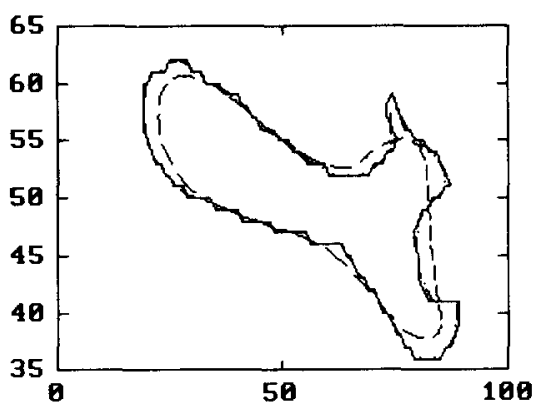

Fig. 4. (a) A wrench and its two moving averages.

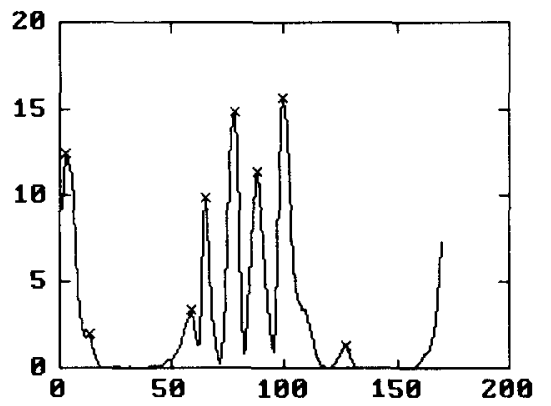

Fig. 4. (b) Local maxima of the absolute difference.

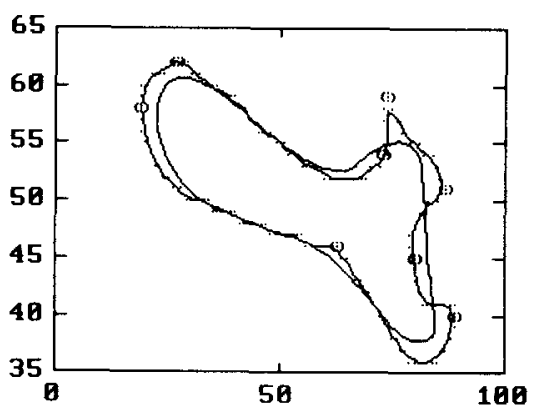

Fig. 4. (c) Corner points.

A simple similar corner point detection system is also examined for comparison. It is a Gaussian convolution mask followed by a curvature local maximum detector. The Gaussian smoothed boundary of a wrench 


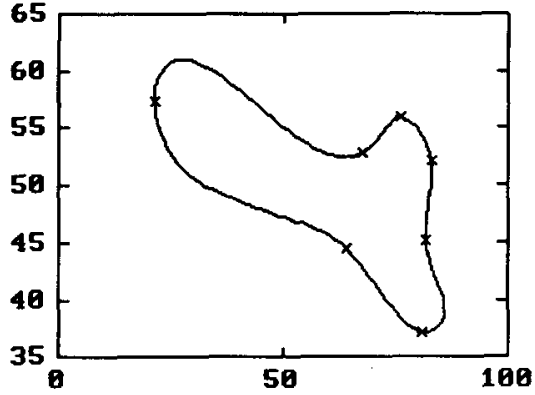

Fig. 5. (a) Curvature local maxima of the Gaussian smoothed boundary.

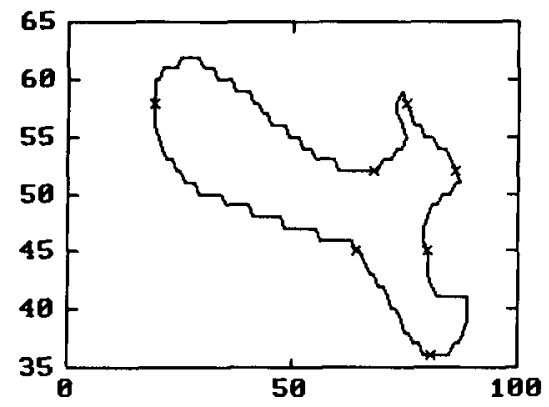

Fig. 5. (b) The positions of the curvature local maxima on the original boundary.

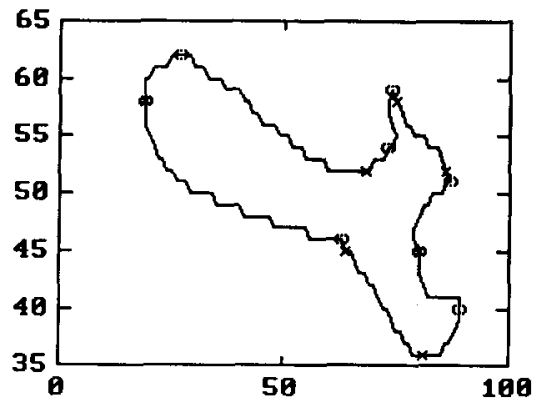

Fig. 5. (c) A comparison between Gaussian smoothing (cross marks) and nest moving average method (small circles).

and the curvature local maximum points are plotted in Fig. 5(a). The positions of the curvature local maximum points on the original boundary are shown in Fig. 5(b). A comparison between the corner point positions detected by this method (indicated by cross marks) and our algorithm (indicated by small circles) is shown in Fig: 5(c). The resulting corner points are similar to those detected by our algorithm, but taking more computing time and suffering from a shift of corner point's position. Although the shifting effect can be eliminated [see reference (6)], the computational complexity will be increased.

\section{DISCUSSION}

The corner detection method we proposed has several advantages

1. Computational efficiency. Convolution is the most time consuming procedure in corner points detection. Our algorithm avoids most of multiplications, thus, high speed convolution is achieved.

2. Locality in spatial domain. The locality property in spatial domain of nest moving average makes it possible for partial shape feature extraction and recognition. Another effect of this property is the further reduction of computational load.

3. Robustness. The corner points are invariant under quantization noise due to the use of small window size moving average.

\section{CONCLUSION}

In this paper, we propose a simple and efficient corner point detection technique. The nest moving average is used as the convolution mask and the curvature local maxima of the resulting shape are extracted as the corners. These methods are invariant under occlusion and rotation; and they are robust under a noisy environment. A fast algorithm for performing the convolution operations is also introduced to reduce the computation time.

In the application to the partial shape recognition, if some part of the shape is occluded or distorted, the nest moving average of the remaining portion will not be affected by the occlusion or distortion.

Using a nest moving average of window size $2 M+1$, only $2 M N$ additions and $2 N$ multiplications are required for performing the convolution of a shape of contour length $N$, the computation is very fast and quite efficient.

\section{REFERENCES}

1. N. Ansari and K.-W. Huang, Non-parametric dominant point detection, Pattern Recognition 24, 849-862 (1991).

2. N. Ansari and E. J. Delp, On detecting dominant points, Pattern Recognition 24, 441-451 (1991).

3. B. Kr. Ray and K. S. Ray, Detection of significant points and polygonal approximation of digitized curves, Pattern Recognition Lett. 13, 443-452 (1992).

4. B. K. Ray and K. S. Ray, An algorithm for detection of dominant points and polygonal approximation of digitized curves, Pattern Recognition Lett. 13, 849-856 (1992).

5. M.-H. Han and D. Jang, The use of maximum curvature points for the recognition of partially occluded objects, Pattern Recognition 23, 21-33 (1990).

6. A. Rattarangsi and R. T. Chin, Scale-based detection of corners of planer curves, IEEE Trans. Pattern Anal. Mach. Intell. 14, 430-449 (April 1992).

7. P. Saint-Marc, J. S. Chen and G. Medioni, Adaptive smoothing: a general tool for early vision, IEEE Trans. Pattern Anal. Mach. Intell. 13, 514-529 (June 1991). 
an engineering officer in the Chinese Navy Shipyard at Peng Fu Island from 1970 to 1971 and a Research Assistant at the University of California, Santa Barbara, from 1971 to 1975. He was Professor and Chairman in the Department of Electrical Engineering at Tatung Institute of Technology from 1981 to 1983. Presently, he is the Professor of the Department of Electrical Engineering at the National Taiwan University. His research interests include digital signal processing, digital picture processing, optical information processing, laser and holography. Dr Pei is a member of the I.E.E.E., Eta Kappa Nu and the Optical Society of America.

About the Author-JI-Hwer HoRng was born in Kinmen, Republic of China, on 13 April 1968. He received the B.S. degree from the Tamkang University in 1990 and the M.S. degree from the National Taiwan University in 1992. Now, he is at the National Taiwan University studying for a Ph.D. degree. His research interests include image analysis and pattern recognition. 